\title{
National plan for clinical guidelines would elevate quality of care, experts say
}

$\mathrm{W}$ ide variations in the quality of Canadian clinical practice guidelines stymies efforts to improve care, as timepressed clinicians must sift through a sea of conflicting recommendations to find pearls of best practice, knowledge translation experts argue.

They're calling for a national strategy to standardize how such guidelines are developed, evaluated and disseminated in a bid to address the inconsistent methodology, lack of collaboration and conflicts of interest that now plagues the landscape. To that end, experts will gather in Gatineau, Quebec to deliberate the steps required for the provinces and territories to pilot a panCanadian clinical guidelines strategy.

"There's this huge knowledge explosion and doctors have difficulty keeping up, so if there's a way of compressing that knowledge into more concise evidence-based recommendations, that would be a huge step forward," explains Dr. Sam Shortt, director of knowledge transfer for the Canadian Medical Association.

Development and implementation of clinical guidelines in Canada is "fragmented" and fraught with difficulty, Shortt says. With no central source to fund or coordinate guideline developers, many work in silos and rely heavily on industry funding (www.cmaj.ca /lookup/doi/10.1503/cmaj.109-3784).

While some recommendations are developed with "very rigorous methodology," many are not, says Patrice Lindsay, performance and standards specialist for the Canadian Stroke Network and a member of the Canadian Task Force on Preventive Health Care. "Different groups will read the same body of evidence and interpret it differently," and most physicians don't have the time to weigh the differences to ensure they're delivering the highest quality care. "We need to get everyone on the same page."

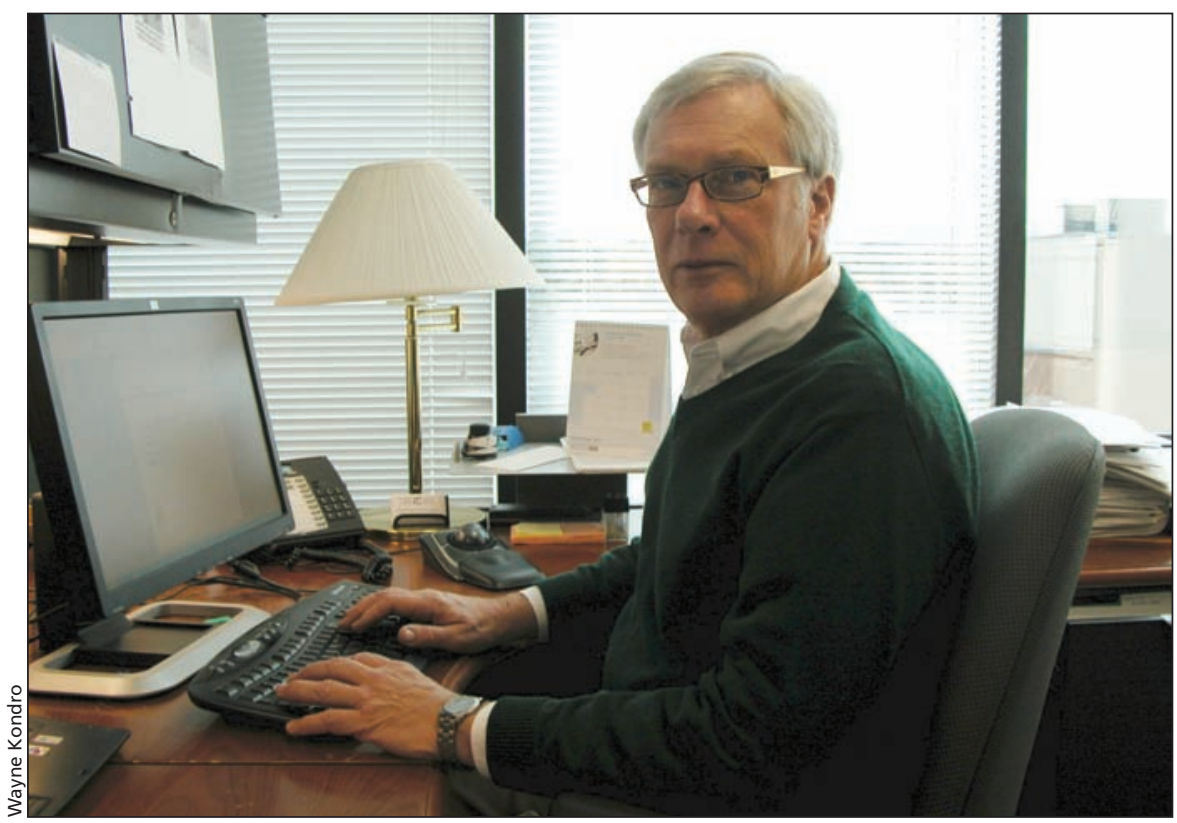

"There's this huge knowledge explosion and doctors have difficulty keeping up, so if there's a way of compressing that knowledge into more concise evidence-based recommendations, that would be a huge step forward," says Dr. Sam Shortt, director of knowledge transfer for the Canadian Medical Association.

Guideline developers are also prone to excessive focus on a single condition, and fail to take into account how their recommendations might fare in the context of treating patients with multiple, complex conditions, says Dr. Ian Graham, vice president of knowledge translation and public outreach for the Canadian Institutes of Health Research.

An ideal national strategy would set standards for the quality and harmonization of guidelines, establish networks for developers to share resources and expertise, and contain accountability measures such as evaluations of the effectiveness and uptake of recommendations, Lindsay says, adding that the latter are essentially missing in Canada.

Physicians often face an array of conflicting guidelines, making them essentially moot, she adds. "When the practitioner doesn't know which one to believe, they'll just go on practicing the way they're most comfortable."
The same goes for overly lengthy or complicated guidelines, says Shortt. "A clinical practice guideline can run 80 pages and that's absolutely no use to someone at point of care."

Several nations, such as the United Kingdom, through its National Institute for Clinical Evidence, have addressed such problems by creating central coordinating bodies to produce or oversee the production of clinical practice guidelines.

But jurisdictional squabbles and a culture of decentralization make it difficult to achieve such a national approach in Canada, Graham says. "Is [Canada] ready for a strategy which will call for collaboration, consensus, [and] cooperation, which may not always benefit individual developers?" he asks.

Finding alternative methods of funding the development of guidelines will be among the biggest challenges, Shortt says. Currently, most guidelines are funded through industry contributions, 
often raising questions of conflict of interest (www.cmaj.ca/lookup/doi/10 .1503/cmaj.109-3757).

"A good guideline is not cheap, although it repays in outcomes for patients, but that's why the major source of funding has been the deep pockets of industry," Shortt says.

Shortt argues that $\$ 10$ million in government funding would provide the infrastructure necessary under a national strategy to oversee the development of guidelines.

Such infrastructure could also develop accountability measures and integrate them into the guidelines to provide physicians and system managers with a mechanism for monitoring implementation, says Lindsay.

"Developing and publishing the guidelines is only a quarter of the process - and we're not even doing that con- sistently," she says. "We need to tell people what to do, show them how to do it, help them advocate to funders to make sure the necessary resources are in place, and then measure, monitor and provide feedback."

The Canadian Clinical Practice Guideline Summit was held Nov. 3-4. — Lauren Vogel, CMAJ

CMAJ 2011. DOI:10.1503/cmaj.109-4043 